УДК 681.518.25:616-77:617.7-76

\title{
СИСТЕМА УПРАВЛЕНИЯ ДВИЖЕНИЯМИ ПАЛЬЦЕВ НА ОСНОВЕ МОДЕЛИ ИСКУССТВЕННОЙ НЕЙРОННОЙ СЕТИ*
}

\author{
К. ВОНСЕВИЧ ${ }^{1 *}$, М. Ф. ГЕТЦЕЛЬ ${ }^{2 * *}$, Е. МРОЗОВСКИ ${ }^{3 * * *}$, Я. АВРЕЙЦЕВИЧ ${ }^{3 * * *}$, М. БЕЗУГЛЫЙ $^{* * * * * *}$ \\ ${ }^{1}$ Национальный технический университет Украины \\ «Киевский политехнический институт им. Игоря Сикорского», \\ Украина, Киев, 03056, пр-т Победы 37 \\ ${ }^{2}$ Университет Сан-Паулу, \\ Бразилия, Сан-Паулу \\ ${ }^{3}$ Лодзинский технический университет, \\ Польша, Лодзь
}

\begin{abstract}
Аннотация. Поверхностный электромиографический (пЭМГ) сигнал применяется в различных сферах, где существует необходимость измерять активность мышц тела человека, например интерфейс «мозг-компьютер», индустрия игр, медицинская техника и другие практические сферы. Более того, использование пЭМГ сигнала в отрасли изготовления активных протезов является традиционным уже в течение многих лет. Однако, несмотря на тот факт, что вопрос о его использовании в сфере протезирования пальцев остается открытым, обычно пЭМГ сигнал требует многоканальных измерительных устройств или массивного объемного оборудования для точного распознания движения кистей рук или пальцев. Это снижает возможную портативность и удобство протезов, и в конечном итоге увеличивает их конечную цену. В этой статье предложен метод организации блока управления и измерения для протеза на основе модели искусственной нейронной сети (ИНС) и пЭМГ измерительной системы на основе одноканального микроконтроллера. Предлагаемая модель ИНС учитывает только 4 входных характеристики пЭМГ сигнала во временной области и обеспечивает точность 95,52\% для классификации 6 различных типов движений пальца. Таким образом, данная модель представляет собой решение, пригодное для реализации в системе протезов пальцев или кисти.
\end{abstract}

Ключевые слова: кисть руки; движения пальцев; электромиография; распознавание образов; искусственная нейронная сеть

\section{1. ВВЕДЕНИЕ}

В повседневной жизни встречаются различные виды взаимодействий между людьми, а также между людьми и предметами. Почти каждый день люди выполняют много различных задач, таких как питье, умывание, приго- товление пищи, употребление пищи, одевание, использование компьютера и т.п. Для успешного осуществления большинства ежедневных занятий (ЕДЗ) движения руки (запястья руки) играют важнейшую роль. Более того, в случае высокоточных задач, таких как писание, наличие запястья и пальцев совершенно необходи-

* Эта работа получила финансовую поддержку от Национального научного центра Польши в рамках гранта OPUS 9 No. 2015/17/B/ST8/01700 на период 2016-2018 годов. 


\section{БИБЛИОГРАФИЧЕСКИЙ СПИСОК}

1. Chien, T.-W.; Lin, W.-S. "Simulation study of activities of daily living functions using online computerized adaptive testing," BMC Med. Inform. Decis. 
Mak., Vol. 16, p. 130-140, 2016. DOI: 10.1186/s12911016-0370-8.

2. Gulde, P.; Hermsdörfer, J. "Both hands at work: the effect of aging on upper-limb kinematics in a multi-step activity of daily living," Exp. Brain Res., Vol. 235, No. 5, p. 1337-1348, 2017. DOI: 10.1007/s00221-017-4897-4.

3. Resnik, L.; Borgia, M.; Acluche, F. "Timed activity performance in persons with upper limb amputation: A preliminary study," J. Hand Ther., Vol. 30, No. 4, p. 468-476, 2017. DOI: 10.1016/j.jht.2017.03.008.

4. Zuniga, J. M.; Carson, A. M.; Peck, J. M.; Kalina, T.; Srivastava, R. M.; Peck, K. "The development of a low-cost three-dimensional printed shoulder, arm, and hand prostheses for children," Prosthet. Orthot. Int., Vol. 41, No. 2, p. 205-209, 2017. DOI: $10.1177 / 03093646166$ 40947.

5. Cordella, F.; Ciancio, A. L.; Sacchetti, R.; Davalli, A.; Cutti, A. G.; Guglielmelli, E.; Zollo, L. "Literature review on needs of upper limb prosthesis users," Front. Neurosci., Vol. 10, p. 1-14, 2016. DOI: 10.3389/fnins. 2016.00209 .

6. Postema, S. G.; Bongers, R. M.; Brouwers, M. A.; Burger, H.; Norling-Hermansson, L. M.; Reneman, M. F.; Dijkstra, P. U.; Van der Sluis, C. K. "Upper limb absence: predictors of work participation and work productivity," Arch. Phys. Med. Rehabil., Vol. 97, p. 892-899, 2016. DOI: $\underline{10.1016 / \mathrm{j} . \mathrm{apmr} .2015 .12 .022 .}$.

7. Burger, H.; Vidmar, G. "A survey of overuse problems in patients with acquired or congenital upper limb deficiency," Prosthet. Orthot. Int., Vol. 40, p. 497-502, 2016. DOI: $10.1177 / 0309364615584658$.

8. Widehammar, C.; Pettersson, I.; Janeslдtt, G.; Hermansson, L. "The influence of environment: Experiences of users of myoelectric arm prosthesis - a qualitative study," Prosthet. Orthot. Int., Vol. 42, No. 1, p. 28-36, 2018. DOI: $10.1177 / 0309364617704801$.

9. Arabian, A.; Varotsis, D.; McDonnell, C.; Meeks, E. "Global social acceptance of prosthetic devices," Proc. of IEEE Glob. Humanit. Technol. Conf., 13-16 Oct 2016, Seattle, USA. IEEE, 2016, p. 563-568. DOI: 10.1109/ GHTC.2016.7857336.

10. Postema, S. G.; Bongers, R. M.; Reneman, M. F.; Van Der Sluis, C. K. "Functional capacity evaluation in upper limb reduction deficiency and amputation: Development and pilot testing," J. Occup. Rehabil., Vol. 28 , No. 1, p. 158-169, 2018. DOI: 10.1007/s10926-0179703-4.

11. Wong, K. V.; Hernandez, A. “A review of additive manufacturing," ISRN Mechanical Engineering, Vol. 2012, ID 208760, p. 1-10, 2012. DOI: $\underline{10.5402 / 2012 / 2}$ $\underline{08760 .}$.

12. Kate, J. T.; Smit, G.; Breedveld, P. "3D-printed upper limb prostheses: a review," Disability and Rehabilitation: Assistive Technology, Vol. 12, No. 3, p. 300-314, 2017. DOI: 10.1080/17483107.2016.1253117.

13. Koprnicky, J.; Najman, P.; Safka, J. “3D printed bionic prosthetic hands," Proc. of 2017 IEEE Int. Workshop on Electronics, Control, Measurement, Signals and their Application to Mechatronics, ECMSM, 24-26 May 2017, Donostia-San Sebastian, Spain. IEEE, 2017, p. 1-6. DOI: $10.1109 /$ ECMSM.2017.7945898.

14. Atzori, M.; Mbller, H. "Control capabilities of myoelectric robotic prostheses by hand amputees: a scientific research and market overview," Front. Syst. Neurosci., Vol. 9, p. 1-7, 2015. DOI: 10.3389/fnsys.2015. 00162.

15. Cowley, B.; Filetti, M.; Lukander, K.; Torniainen, J.; Henelius, A.; Ahonen, L.; Barral, O.; Kosunen, I.; Valtonen, T.; Huotilainen, M.; Ravaja, N.; Jacucci, G. "The psychophysiology primer: a guide to methods and a broad review with a focus on human-computer interaction," in: Foundations and Trends in Human-Computer Interaction, 2016, Vol. 9, No. 3-4, p. $150-307$. DOI: $10.1561 / 1100000065$.

16. Ma, W.; Zhang, X.; Yin, G. "Design on intelligent perception system for lower limb rehabilitation exoskeleton robot," Proc. of IEEE 13th Int. Conf. on Ubiquitous Robot and Ambient Intelligence, 19-22 Aug 2016, Xian, China. IEEE, 2016, p. 587-592. DOI: 10.1109/URAI.2016.7625785.

17. Sharma, S.; Farooq, H.; Chahal, N. "Feature extraction and classification of surface EMG signals for robotic hand simulation," Commun. Appl. Electron., Vol. 4, p. 27-31, 2016. DOI: 10.5120/cae2016652042.

18. Spanias, J. A.; Perreault, E. J.; Hargrove, L. J. "Detection of and compensation for EMG disturbances for powered lower limb prosthesis control," IEEE Trans. Neural Syst. Rehabil. Eng., Vol. 24, No. 2, p. 226-234, 2016. DOI: $10.1109 /$ TNSRE.2015.2413393.

19. Gailey, A.; Artemiadis, P.; Santello, M. "Proof of concept of an online EMG-based decoding of hand postures and individual digit forces for prosthetic hand control," Front. Neurol., Vol. 8, p. 1-15, 2017. DOI: 10.3389/fneur. 2017.00007.

20. Na, Y.; Kim, S. J.; Jo, S.; Kim, J. "Ranking hand movements for myoelectric pattern recognition considering forearm muscle structure," Med. Biol. Eng. Comput., Vol. 55, No. 8, p. 1507-1518, 2017. DOI: 10.1007/s11517016-1608-4.

21. Ariyanto, M.; Caesarendra, W.; Mustaqim, K. A.; Irfan M.; Pakpahan, J. A.; Setiawan J. D.; Winoto, A. R. "Finger movement pattern recognition method using artificial neural network based on electromyography (EMG) sensor," Proc. of Int. Conf. on Automation, Cognitive Science, Optics, Micro Electro-Mechanical System, and Information Technology, ICACOMIT, 29-30 Oct 2015, Bandung, Indonesia. IEEE, 2015, p. 12-17. DOI: $10.1109 /$ ICACOMIT.2015.7440146.

22. Tenore, F. V. G.; Ramos, A.; Fahmy, A.; Acharya, S.; Etienne-Cummings, R.; Thakor N. V. "Decoding of individuated finger movements using surface electromyography," IEEE Trans. Biomed. Eng., Vol. 56, No. 5, p. 1427-1434, 2009. DOI: 10.1109/TBME. 2008.2005485 .

23. Zecca, M.; Micera, S.; Carrozza, M. C.; Dario, P. "Control of multifunctional prosthetic hands by processi- 
ng the electromyographic signal," Crit. Rev. Biomed. Eng., Vol. 30, No. 4-6, p. 459-485, 2002. DOI: 10.16 15/CritRevBiomedEng.v30.i456.80.

24. Micera, S.; Carpaneto, J.; Raspopovic, S. "Control of hand prosthesis using peripheral information," IEEE Rev. Biomed. Eng., Vol. 3, p. 48-68, 2010. DOI: 10.1109/RBME.2010.2085429.

25. Strazzulla, I.; Nowak, M.; Controzzi, M.; Cipriani, C.; Castellini, C. "Online bimanual manipulation using surface electromyography and incremental learning," IEEE Trans. Neural Syst. Rehabil. Eng., Vol. 25, No. 3, p. 227-234, 2017. DOI: 10.1109/TNSRE.2016. 2554884 .

26. Tavakoli, M.; Benussi, C.; Lourenco, J. L. "Single channel surface EMG control of advanced prosthetic hands: A simple, low cost and efficient approach," Expert Syst. Appl., Vol. 79, p. 322-332, 2017. DOI: $10.1016 /$ j.eswa.2017.03.012.

27. Вонсевич, К.П.; Безуглий, М.О.; Гапонюк, А. О. "Інформаційно-вимірювальна система міографу біонічного протезу кінцівки," Перспективні технологї та прилади, Т. 10, С. 32-37, 2017.

28. Heiderich, M.; Leonhardt, S.; Krantz, W.; Neubeck, J.; Wiedemann, J. "Method for analysing the feeling of safety at high speed using virtual test drives," Proc. of 18 Internationales Stuttgarter Symp., Wiesbaden:
Springer Vieweg, 2018, p. 875-886. DOI: $10.1007 / 978-$ 3-658-21194-3 67.

29. Horwitz A. "A version of Simpson's rule for multiple integrals," J. Computational Applied Math., Vol. 134, No. 1-2, p. 1-11, 2001. DOI: 10.1016/S0377-0427 (00)00444-1.

30. Levenberg, K. "A method for the solution of certain non-linear problems in least squares," $Q$. Appl. Math., Vol. 2, No. 2, p. 164-168, 1944. URI: https://www. jstor.org/stable/43633451.

31. Marquardt, D. W. "An algorithm for least-squares estimation of nonlinear parameters," J. Soc. Ind. Appl. Math., Vol. 11, No. 2, p. 431-441, 1963. DOI: $10.1137 /$ 0111030 .

32. Rumelhart, D. E.; Hinton, G. E.; Williams, R. J. "Learning representations by back-propagating errors," Nature, Vol. 323, p. 533-536, 1986. DOI: $\underline{10.1038 / 3235}$ $\underline{33 \mathrm{a} 0}$.

33. Swets, J. A. "Measuring the accuracy of diagnostic systems," Science, Vol. 240, No. 4857, p. 1285-1293, 1988. DOI: $10.1126 /$ science. 3287615 .

34. Kim, S.; Kim, J.; Ahn, S.; Kim, Y. "Finger language recognition based on ensemble artificial neural network learning using armband EMG sensors," Technology Health Care, Vol. 26, No. S1, p. 249-258, 2018. DOI: $10.3233 /$ THC-174602. 